\title{
Association between breakfast skipping and metabolic outcomes by sex, age, and work status stratification
}

\author{
Jun Heo ${ }^{1}$, Won-Jun $\mathrm{Choi}^{2}$, Seunghon Ham², Seong-Kyu Kang ${ }^{2}$ and Wanhyung Lee ${ }^{2^{*}}$ (D)
}

\begin{abstract}
Background: The association between breakfast skipping and abnormal metabolic outcomes remains controversial. A comprehensive study with various stratified data is required.

Objective: The aim of this study was to investigate the relationship between abnormal metabolic outcomes and breakfast skipping by sex, age, and work status stratification.

Methods: We used data from the Korea National Health and Nutrition Examination Surveys from 2013 to 2018. A total of 21,193 (9022 men and 12,171 women) participants were included in the final analysis. The risk of metabolic outcomes linked to breakfast skipping was estimated using the negative binomial regression analysis by sex, work status, and age stratification.

Results: A total of 11,952 (56.4\%) participants consumed breakfast regularly. The prevalence of abnormal metabolic outcomes was higher among those with irregular breakfast consumption habits. Among young male workers, negative binomial regression analysis showed that irregular breakfast eaters had a higher risk of abnormal metabolic outcomes, after adjusting for covariates (odds ratio, 1.15; 95\% confidence interval, 1.03-1.27).

Conclusions: The risk of abnormal metabolic outcomes was significant in young men in the working population. Further studies are required to understand the association of specific working conditions (working hours or shift work) with breakfast intake status and the risk of metabolic diseases.
\end{abstract}

Keywords: Breakfast skipping, Abnormal metabolic outcomes, Working conditions

\section{Introduction}

Breakfast is the most important meal of the day because it helps the human body to start daily metabolism. The human body is regulated by circadian rhythms. Circadian rhythms are influenced by the light-dark cycle, as well as by food uptake, which is the metabolic signal. Inversely, circadian regulation of metabolic genes affects metabolic outcomes in the human body, which signifies that feeding

\footnotetext{
${ }^{*}$ Correspondence: wanhyung@gmail.com

2 Department of Occupational and Environmental Medicine, Gil Medical Center, Gachon University College of Medicine, 21, Namdong-daero 774 beon-gil, Namdong-gu, Incheon 21565, Republic of Korea

Full list of author information is available at the end of the article
}

time and the circadian clock are tightly intertwined [1]. Breakfast is important to jumpstart daily metabolism. A randomized clinical trial showed that breakfast skipping adversely affected circadian gene expression and correlated with increased postprandial glycemic response [2]. The irregular consumption of breakfast can induce various health problems.

Many studies have reported the association between breakfast skipping and health problems. A large, prospective study conducted in the US on middle-aged and older male health professionals in the US confirmed that eating breakfast was associated with a significantly lower risk of coronary heart disease [3]. Some 
studies found that individuals who skipped breakfast had higher rates of mortality [4], higher serum cholesterol levels [5], and frequent health-compromising behaviors [6], compared with regular breakfast eaters. In addition, other studies have reported that breakfast intake has many beneficial effects such as improved satiety, reduced incidence of food cravings [7] and improved cognitive function and academic performance [8].

Breakfast skipping has a significant impact on body weight and metabolic outcomes. The relationship between breakfast skipping and high body mass index (BMI) values has been widely reported in adolescent populations in Europe [9], Hong Kong [10], and Fiji (girls) [11]. Similar associations were reported in the adult [12], middle-aged adult [13], and elderly [14] populations. Many studies have reported the association between metabolic outcomes and breakfast skipping; however, more evidence is required. The strength of the association between breakfast habituation and metabolic outcomes varies according to age group, sex, and ethnicity. For instance, a cross-sectional study on 5316 American young adults showed that regular breakfast eaters were less likely to have elevated low-density lipoprotein cholesterol (LDL-C) levels, high blood pressure, and reduced serum high-density lipoprotein cholesterol (HDL-C) levels [15]. In contrast, a study on 415 Korean adults confirmed that regular breakfast intake was associated with elevated triglyceride (TG) levels [16]. Thus, studies on the association between breakfast skipping and metabolic syndrome remain conflicting, warranting further studies on this subject.

This study was undertaken to (1) identify the relationship between breakfast skipping and metabolic outcomes in the Korean adult population and (2) demonstrate, in detail, the effect of breakfast skipping on metabolic outcomes according to age group, sex, and work status.

\section{Methods}

\section{Data and study participants}

We used data from the Korea National Health and Nutrition Examination Surveys (KNHANES) from 2013 to 2018. KNHANES, which has been conducted every year since 1998 by the Korea Centers for Disease Control and Prevention (KCDC), is a series of nationally representative, population-based surveys on the health and nutritional status of Korean citizens [17]. The KNHANES database is publicly available on their website (http:// knhanes.cdc.go.kr, available in Korean). More than 7000 participants were selected each year by the stratified random sampling method. Data were collected through interviews, blood tests, urine tests, and physical examinations in the examination vehicle. KNHANES was approved by the KCDC Institutional Review Board, and all participants provided written informed consent. The total number of participants in KNHANES from 2013 to 2018 was 47,217 . We excluded the following participants: (1) those older than 59 years or less than 20 years of age $(n=16,394)$; (2) those who refused to answer questions regarding their work status $(n=5063)$; and (3) those with missing information for metabolic outcomes, frequency of breakfast consumption, education level, and household income $(n=4567)$. After all the exclusions, the final number of participants included in this analysis was 21,193 (9022 men and 12,171 women, Fig. 1).

\section{Status of breakfast consumption}

The frequency of breakfast consumption was assessed using a self-administered questionnaire. Participants were asked to report the average number of breakfasts consumed per week in the past year; four categories were considered: 5-7/week, 3-4/week, 1-2/week, and 0/week. We then categorized the participants into two groups: (1) regular breakfast eaters (those who ate breakfast almost every day; 5-7/week category) and (2) irregular breakfast
The Korea National Health and Nutrition Examination Surveys (KNHANES) in 2013-2018 $\mathrm{n}=47,217$

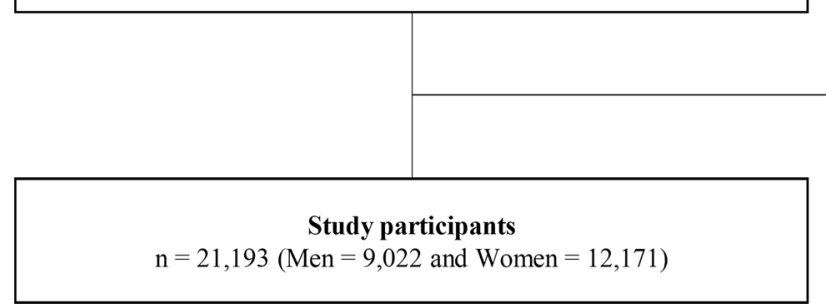

Fig. 1 Schematic diagram depicting the study population

\begin{tabular}{|c|}
\hline Exclusion \\
\hline Non-target aged population $(\mathrm{n}=16,394)$ \\
Missing information $(\mathrm{n}=5,063)$ \\
Refusal data $(\mathrm{n}=4,567)$ \\
\hline
\end{tabular}


eaters (those who ate breakfast rarely or never; 3-4/week, $1-2 /$ week, and $0 /$ week categories).

\section{Measurement of metabolic outcomes}

Metabolic outcomes included central obesity, raised blood pressure, raised fasting serum glucose, increased TG, and decreased HDL-C levels from definition of metabolic syndrome according to the National Cholesterol Education Program Adult Treatment Panel III (NCEP-ATP III) criteria [18]. Abnormal metabolic outcomes were defined as follows: (i) central obesity: waist circumference (WC) $\geq 90 \mathrm{~cm}$ in men, and $\mathrm{WC} \geq 80 \mathrm{~cm}$ in women, in line with the Asian standard; (ii) raised blood pressure: systolic blood pressure $\geq 130 \mathrm{mmHg}$, diastolic blood pressure $\geq 85 \mathrm{mmHg}$, or pharmacologic treatment for hypertension; (iii) raised fasting serum glucose: fasting serum glucose $\geq 100 \mathrm{mg} / \mathrm{dL}$ or pharmacologic treatment for diabetes; (iv) elevated TG levels: serum TG $\geq 150 \mathrm{mg} / \mathrm{dL}$ or pharmacologic treatment for dyslipidemia; and (v) decreased HDL-C: levels of $<40 \mathrm{mg} / \mathrm{dL}$ in men and $<50 \mathrm{mg} / \mathrm{dL}$ in women or pharmacologic treatment for dyslipidemia. The presence of three or more is defined as metabolic syndrome.

Blood pressure was measured following the standard protocol using a mercury manometer [19]. Serum glucose, TG, and HDL-C levels were measured on a Hitachi 7600-210 automatic analyzer (Hitachi, Japan), using the hexokinase UV, enzymatic, and homogeneous enzymatic colorimetric methods, respectively.

\section{Covariates}

We considered age, education, household income, smoking, alcohol drinking, and physical activity as covariates. Workers were defined as a paid working group to reduce the heterogeneity of work characteristics. We classified education into three categories based on the highest level of education as follows: (1) below middle school, (2) high school, and (3) university. Household income was divided into four quartiles. Smoking status was divided into three categories (current, former, and never). Alcohol intake was classified into three categories (severe, moderate, and none). Physical activity was defined as "yes" if the participant performed vigorous-intensity physical activity for at least $75 \mathrm{~min}$, moderate-intensity physical activity for at least $150 \mathrm{~min}$, or an equivalent combination of moderate and vigorous activity per week [20].

\section{Statistical analyses}

All statistical analyses were performed using Statistical Analysis System version 9.4 (SAS Institute, Cary, NC, USA). Chi-square tests were conducted to assess the differences in general characteristics based on regular breakfast intake. Student t-tests were conducted to compare the average of each measurement of metabolic outcomes and the total number of abnormal metabolic outcomes, based on regular breakfast intake. Weighted prevalence of number of metabolic outcomes by breakfast consumption were calculated using the KNHANES sample weights which were constructed for sample participants to represent the Korean population by accounting for the complex survey design, survey non-response, and post-stratification. Number of abnormal metabolic outcomes showed non-normal distributions in the current study. The negative binomial model was selected to appropriate regression model considering Akaike information criteria and Bayesian information criteria, which are the criteria used for assessing model goodness of fit compared with Poisson or zero-inflated negative binominal model. Multivariate negative binominal regressions were used to explore the association between the risk of increasing number of abnormal metabolic outcomes and regularity of breakfast intake, with adjustments for age, education level, income level, smoking, alcohol intake, and physical activity according to sex, work status, and age stratification, to estimate the odds ratio (OR) and 95\% confidence intervals (CIs).

\section{Results}

The baseline characteristics according to regular breakfast intake are presented in Table 1. The total number of participants was 21,193 (9022 men and 12,171 women), and $11,952(56.4 \%)$ were regular breakfast eaters. No significant difference was observed in regular breakfast intake between men and women. The participants were stratified based on age as young (age, 20-39years) and middle-aged (age, 40-59 years). Among regular breakfast eaters, the percentage of young participants $(n=2775$, 23.2\%) was significantly lower than that of middle-aged participants $(n=9177,76.8 \%)$. The proportion of participants with a high level of education was higher among irregular breakfast eaters. In contrast, the proportion of participants with a larger household income was higher among regular breakfast eaters.

The percentage of those with unhealthy lifestyle habits was higher among irregular breakfast eaters, except for physical activity. In our study, $13.1 \%$ of regular breakfast eaters, compared with $22.5 \%$ of irregular breakfast eaters, were current smokers. Furthermore, only $8.9 \%$ of regular breakfast eaters were heavy drinkers, compared with $14.1 \%$ of the irregular breakfast eaters. There was no significant difference in physical activity level between regular breakfast and irregular breakfast eaters. Individuals with abnormal metabolic outcomes, except abdominal obesity, significantly tend to demonstrate regular breakfast consumption habits. The specific metabolic outcomes stratified by working population are shown in 
Table 1 General characteristics of study participants by breakfast consumption

\begin{tabular}{|c|c|c|c|}
\hline \multirow[t]{2}{*}{ Characteristics } & \multicolumn{2}{|c|}{ Regular breakfast, n (\%) } & \multirow[t]{2}{*}{$p$-value } \\
\hline & Yes & No & \\
\hline Total & $11,952(56.4)$ & $9241(43.6)$ & \\
\hline Sex & & & 0.1100 \\
\hline Men & $5031(42.1)$ & $3991(43.2)$ & \\
\hline Women & $6921(57.9)$ & $5250(56.8)$ & \\
\hline Age & & & $<.0001$ \\
\hline $20-39$ & $2775(23.2)$ & $4544(49.2)$ & \\
\hline $40-59$ & $9177(76.8)$ & $4697(50.8)$ & \\
\hline Education & & & $<.0001$ \\
\hline Middle school & $3671(30.7)$ & $1707(18.5)$ & \\
\hline High school & $3878(32.5)$ & $3435(37.2)$ & \\
\hline College or more & $4403(36.8)$ & 4099 (44.4) & \\
\hline Household income & & & $<.0001$ \\
\hline 1st Quartile & $942(7.9)$ & $821(8.9)$ & \\
\hline 2nd Quartile & $2707(22.7)$ & $2278(24.6)$ & \\
\hline 3rd Quartile & $3785(31.7)$ & $3086(33.4)$ & \\
\hline 4th Quartile & $4518(37.8)$ & $3056(33.1)$ & \\
\hline Smoking & & & $<.0001$ \\
\hline None & $8636(72.3)$ & $5856(63.4)$ & \\
\hline Past & $1754(14.7)$ & $1304(14.1)$ & \\
\hline Current & $1562(13.1)$ & $2081(22.5)$ & \\
\hline Drinking & & & $<.0001$ \\
\hline None & $4122(34.6)$ & $2105(22.8)$ & \\
\hline Moderate & $6743(56.5)$ & $5829(63.1)$ & \\
\hline Severe & $1062(8.9)$ & $1300(14.1)$ & \\
\hline Physical activity & & & 0.0008 \\
\hline No or unknown & $7304(61.1)$ & $5438(58.9)$ & \\
\hline Yes & $4648(38.9)$ & $3803(41.2)$ & \\
\hline Working & & & $<.0001$ \\
\hline No & $7042(58.9)$ & $4754(51.4)$ & \\
\hline Yes & $4910(41.1)$ & 4487 (48.6) & \\
\hline Abdominal obesity & & & 0.4420 \\
\hline No & $9601(80.3)$ & 7384 (79.9) & \\
\hline Yes & $2351(19.7)$ & $1857(20.1)$ & \\
\hline Raised blood pressure & & & $<.0001$ \\
\hline No & $8954(74.9)$ & 7305 (79.1) & \\
\hline Yes & $2998(25.1)$ & $1936(20.9)$ & \\
\hline Raised fasting glucose & & & $<.0001$ \\
\hline No & $8932(74.7)$ & $7128(77.1)$ & \\
\hline Yes & $3020(25.3)$ & $2113(22.9)$ & \\
\hline Increased TG & & & 0.0195 \\
\hline No & $8674(72.6)$ & $6839(74.1)$ & \\
\hline Yes & $3278(27.4)$ & $2402(26.9)$ & \\
\hline Decreased HDL-C & & & $<.0001$ \\
\hline No & 7877 (65.9) & 6428 (69.6) & \\
\hline Yes & $4075(34.1)$ & $2813(30.4)$ & \\
\hline Metabolic syndrome & & & $<.0001$ \\
\hline No & $9523(79.7)$ & 7617 (82.4) & \\
\hline
\end{tabular}

Table 1 (continued)

\begin{tabular}{llll}
\hline Characteristics & \multicolumn{2}{l}{ Regular breakfast, $\mathbf{n}(\%)$} & p-value \\
\cline { 2 - 3 } & Yes & No & \\
\hline Yes & $2429(20.3)$ & $1624(17.6)$ \\
\hline
\end{tabular}

Abdominal obesity: $W C>90 \mathrm{~cm}$ for male, $W C>80 \mathrm{~cm}$ for female (Asian modified) Raised blood pressure: Systolic blood pressure $\geq 130 \mathrm{mmHg}$, Diastolic blood pressure $\geq 85 \mathrm{mmHg}$, or pharmacologic treatment for hypertension

Raised fasting plasma glucose: Plasma fasting glucose $\geq 100 \mathrm{mg} / \mathrm{dL}$ or pharmacologic treatment for diabetes

Increased triglyceride(TG): Plasma triglyceride $\geq 150 \mathrm{mg} / \mathrm{dL}$ or pharmacologic treatment for dyslipidemia

Decreased high density lipoprotein cholesterol(HDL-C): Plasma HDL-C $<40 \mathrm{mg} /$ $\mathrm{dL}$ for male, Plasma-HDL-C $<50 \mathrm{mg} / \mathrm{dL}$ for female or pharmacologic treatment for dyslipidemia

Metabolic syndrome: Three or more of the above traits (NCEP-ATP III Criteria)

Table 2. We considered the average value of each metabolic outcome according to the breakfast consumption status. Age was not stratified in the above analysis.

The weighted prevalence of abnormal metabolic outcome according to breakfast consumption status, stratified by age, sex, and working status is presented in Table 3. After stratification of sex, age, and working status, there was a significant difference in weighted prevalence between regular and irregular breakfast eaters. Among young male workers, 41.0 and $34.9 \%$ of regular and irregular breakfast eaters, respectively, had normal metabolic outcomes, with zero metabolic abnormality. In contrast, in the middle-aged female worker group, regular breakfast eaters had a significantly higher number of metabolic abnormalities.

The association between metabolic abnormalities and irregular breakfast consumption after adjusting for age, education, household income, smoking status, alcohol drinking status, and physical activity is presented in Table 4. Negative binomial regression analysis revealed that an irregular breakfast group had a higher risk of increased number of metabolic abnormalities in the younger working men population than regular breakfast group (odds ratio, 1.15; 95\% confidence interval, 1.031.27). There was no significant association between the number of metabolic abnormalities and irregular breakfast consumption in middle-aged female workers after adjustment.

\section{Discussion}

We conducted this study to understand the association between regular breakfast intake and metabolic outcomes by sex, work status, and age group stratification. The breakfast consumption pattern was consistent with previous studies. Of the total participants, $56.4 \%$ consumed breakfast regularly. Similar patterns was observed 
Table 2 Metabolic outcomes by breakfast consumption

\begin{tabular}{|c|c|c|c|}
\hline \multirow[t]{2}{*}{ Characteristics } & \multicolumn{2}{|c|}{ Regular breakfast, mean (standard deviation) } & \multirow[t]{2}{*}{ p-value } \\
\hline & Yes & No & \\
\hline \multicolumn{4}{|l|}{ Total participants } \\
\hline \multicolumn{4}{|l|}{ Men } \\
\hline Abdominal circumference $(\mathrm{cm})$ & $82.53(11.2)$ & $83.83(10.8)$ & 0.0083 \\
\hline Systolic blood pressure $(\mathrm{mmHg})$ & $116.7(13.7)$ & $116.8(12.7)$ & 0.9456 \\
\hline Diastolic blood pressure $(\mathrm{mmHg})$ & $76.32(11.5)$ & $77.53(10.9)$ & $<.0001$ \\
\hline Fasting glucose level (mg/dL) & $99.74(22.5)$ & $98.05(21.2)$ & 0.0003 \\
\hline TG level (mg/dL) & $149.6(13.7)$ & $156.8(13.8)$ & 0.0128 \\
\hline HDL-C level (mg/dL) & $48.14(10.9)$ & $47.95(10.9)$ & 0.3939 \\
\hline Number of abnormal metabolic outcomes & $1.52(1.5)$ & $1.51(1.4)$ & 0.7491 \\
\hline \multicolumn{4}{|l|}{ Women } \\
\hline Abdominal circumference $(\mathrm{cm})$ & $75.69(9.6)$ & $75.30(9.7)$ & 0.0285 \\
\hline Systolic blood pressure $(\mathrm{mmHg})$ & $111.2(14.8)$ & $109.0(13.3)$ & $<.0001$ \\
\hline Diastolic blood pressure $(\mathrm{mmHg})$ & $72.47(9.9)$ & $72.15(9.5)$ & 0.0662 \\
\hline Fasting glucose level (mg/dL) & $94.78(18.1)$ & $93.68(18.2)$ & 0.0010 \\
\hline TG level (mg/dL) & $105.9(79.5)$ & $101.7(73.9)$ & 0.0028 \\
\hline HDL-C level (mg/dL) & $55.06(12.2)$ & $56.30(12.3)$ & $<.0001$ \\
\hline Number of abnormal metabolic outcomes & $1.17(1.3)$ & $0.97(1.2)$ & $<.0001$ \\
\hline \multicolumn{4}{|l|}{ Non-working population } \\
\hline \multicolumn{4}{|l|}{ Men } \\
\hline Abdominal circumference $(\mathrm{cm})$ & $80.18(12.5)$ & $81.96(11.8)$ & $<.0001$ \\
\hline Systolic blood pressure $(\mathrm{mmHg})$ & $115.4(13.5)$ & $116.2(13.0)$ & 0.0306 \\
\hline Diastolic blood pressure $(\mathrm{mmHg})$ & $73.47(12.2)$ & $75.61(11.4)$ & $<.0001$ \\
\hline Fasting glucose level (mg/dL) & $98.98(21.6)$ & $97.84(22.2)$ & 0.0841 \\
\hline TG level (mg/dL) & $136.7(12.5)$ & $148.0(13.5)$ & 0.0042 \\
\hline HDL-C level (mg/dL) & $48.47(10.9)$ & $48.45(10.8)$ & 0.9474 \\
\hline Number of abnormal metabolic outcomes & $1.34(1.4)$ & $1.36(1.4)$ & 0.6741 \\
\hline \multicolumn{4}{|l|}{ Women } \\
\hline Abdominal circumference $(\mathrm{cm})$ & $75.19(10.0)$ & $75.41(10.2)$ & 0.3559 \\
\hline Systolic blood pressure $(\mathrm{mmHg})$ & $110.9(14.7)$ & $109.0(13.3)$ & $<.0001$ \\
\hline Diastolic blood pressure $(\mathrm{mmHg})$ & $71.89(10.1)$ & $71.87(9.6)$ & 0.9338 \\
\hline Fasting glucose level (mg/dL) & $94.58(17.5)$ & $94.20(20.1)$ & 0.4166 \\
\hline TG level (mg/dL) & $107.7(77.0)$ & $104.2(72.1)$ & 0.0500 \\
\hline HDL-C level (mg/dL) & $54.51(11.9)$ & $55.68(12.3)$ & $<.0001$ \\
\hline Number of abnormal metabolic outcomes & $1.18(1.3)$ & $1.03(1.2)$ & $<.0001$ \\
\hline \multicolumn{4}{|l|}{ Working population } \\
\hline \multicolumn{4}{|l|}{ Men } \\
\hline Abdominal circumference $(\mathrm{cm})$ & $85.27(8.7)$ & $85.40(9.5)$ & 0.6219 \\
\hline Systolic blood pressure $(\mathrm{mmHg})$ & $118.3(13.8)$ & $117.2(12.4)$ & 0.0035 \\
\hline Diastolic blood pressure $(\mathrm{mmHg})$ & $79.65(9.8)$ & $79.14(10.2)$ & 0.0925 \\
\hline Fasting glucose level (mg/dL) & $100.6(23.5)$ & $98.23(20.4)$ & 0.0003 \\
\hline TG level (mg/dL) & $164.7(14.8)$ & $164.3(14.0)$ & 0.9257 \\
\hline HDL-C level (mg/dL) & $47.77(10.8)$ & $47.53(10.9)$ & 0.4624 \\
\hline Number of abnormal metabolic outcomes & $1.72(1.4)$ & $1.63(1.4)$ & 0.0375 \\
\hline \multicolumn{4}{|l|}{ Women } \\
\hline Abdominal circumference $(\mathrm{cm})$ & $76.53(8.9)$ & $75.16(9.1)$ & $<.0001$ \\
\hline Systolic blood pressure (mmHg) & $111.7(14.9)$ & $108.9(13.2)$ & $<.0001$ \\
\hline Diastolic blood pressure $(\mathrm{mmHg})$ & $73.45(9.6)$ & $72.49(9.3)$ & 0.0004 \\
\hline Fasting glucose level (mg/dL) & $95.12(19.1)$ & $93.02(15.4)$ & $<.0001$ \\
\hline TG level (mg/dL) & $102.9(83.5)$ & $98.56(76.0)$ & 0.0580 \\
\hline HDL-C level (mg/dL) & $56.00(12.4)$ & $57.09(12.4)$ & 0.0021 \\
\hline Number of abnormal metabolic outcomes & $1.16(1.3)$ & $0.91(1.1)$ & $<.0001$ \\
\hline
\end{tabular}


Table 3 Weighted prevalence of abnormal metabolic outcome by breakfast consumption

\begin{tabular}{|c|c|c|c|c|c|c|c|}
\hline & \multicolumn{6}{|c|}{ Number of metabolic abnormalities, Regular / Irregular breakfast, \% of row (standard errors) } & \multirow[t]{2}{*}{ p-value } \\
\hline & 0 & 1 & 2 & 3 & 4 & 5 & \\
\hline $\begin{array}{l}\text { Total participants } \\
(\mathrm{n}=27,627,602)\end{array}$ & $\begin{array}{l}37.3(0.4) / 40.7 \\
(0.3)\end{array}$ & $\begin{array}{l}25.6(0.3) / 25.8 \\
(0.3)\end{array}$ & $\begin{array}{l}16.8(0.2) / 15.9 \\
(0.2)\end{array}$ & $\begin{array}{l}11.0(0.2) / 10.1 \\
(0.2)\end{array}$ & $6.9(0.1) / 5.3(0.1)$ & $2.3(0.1) / 2.1(0.1)$ & $<.0001$ \\
\hline $\begin{array}{l}\text { Men } \\
\quad(n=13,597,358)\end{array}$ & $\begin{array}{l}32.6(0.5) / 33.0 \\
(0.5)\end{array}$ & $\begin{array}{l}22.9(0.4) / 24.2 \\
(0.3)\end{array}$ & $\begin{array}{l}18.9(0.3) / 18.7 \\
(0.3)\end{array}$ & $\begin{array}{l}13.4(0.3) / 13.5 \\
(0.3)\end{array}$ & $9.0(0.2) / 7.6(0.2)$ & $3.2(0.1) / 3.0(0.1)$ & 0.3096 \\
\hline $\begin{array}{c}\text { Younger aged Men } \\
(n=5,534,982)\end{array}$ & $\begin{array}{l}42.2(0.8) / 36.1 \\
(0.8)\end{array}$ & $\begin{array}{l}25.4(0.6) / 26.0 \\
(0.7)\end{array}$ & $\begin{array}{l}17.6(0.5) / 18.0 \\
(0.7)\end{array}$ & $8.7(0.3) / 11.6(0.5)$ & $5.1(0.3) / 6.3(0.4)$ & $1.0(0.1) / 2.0(0.2)$ & 0.0067 \\
\hline $\begin{array}{l}\text { Middle aged Men } \\
\quad(n=8,062,377)\end{array}$ & $\begin{array}{l}29.1(0.6) / 29.1 \\
(0.5)\end{array}$ & $\begin{array}{l}22.0(0.5) / 21.8 \\
(0.4)\end{array}$ & $\begin{array}{l}19.4(0.5) / 19.6 \\
(0.4)\end{array}$ & $\begin{array}{l}15.1(0.4) / 15.9 \\
(0.4)\end{array}$ & $10.4(0.4) / 9.2(0.3)$ & $4.0(0.2) / 4.3(0.2)$ & 0.8014 \\
\hline $\begin{array}{l}\text { Women } \\
\qquad(n=14,030,244)\end{array}$ & $\begin{array}{l}41.8(0.5) / 48.5 \\
(0.5)\end{array}$ & $\begin{array}{l}28.2(0.4) / 27.5 \\
(0.4)\end{array}$ & $\begin{array}{l}14.8(0.3) / 13.0 \\
(0.3)\end{array}$ & $8.8(0.2) / 6.6(0.2)$ & $5.1(0.2) / 3.1(0.1)$ & $1.5(0.1) / 1.2(0.1)$ & $<.0001$ \\
\hline $\begin{array}{l}\text { Younger aged } \\
\text { Women } \\
(n=5,408,303)\end{array}$ & $\begin{array}{l}58.1(0.7) \\
/ 57.4(0.9)\end{array}$ & $\begin{array}{l}26.6(0.5) \\
/ 26.3(0.6)\end{array}$ & $\begin{array}{l}10.3(0.3) \\
/ 10.6(0.4)\end{array}$ & $\begin{array}{l}3.1(0.2) \\
/ 3.9(0.3)\end{array}$ & $\begin{array}{l}1.3(0.1) \\
/ 1.3(0.1)\end{array}$ & $\begin{array}{l}0.6(0.1) \\
/ 0.5(0.1)\end{array}$ & 0.8674 \\
\hline $\begin{array}{l}\text { Middle aged } \\
\text { Women } \\
(n=8,621,941)\end{array}$ & $\begin{array}{l}35.8(0.6) \\
/ 38.7(0.5)\end{array}$ & $\begin{array}{l}28.7(0.5) \\
/ 28.9(0.4)\end{array}$ & $\begin{array}{l}16.4(0.4) \\
/ 15.7(0.3)\end{array}$ & $\begin{array}{l}10.9(0.3) \\
/ 9.6(0.2)\end{array}$ & $\begin{array}{l}6.4(0.2) \\
/ 5.0(0.2)\end{array}$ & $\begin{array}{l}1.8(0.1) \\
/ 2.0(0.1)\end{array}$ & 0.0516 \\
\hline $\begin{array}{l}\text { Non-working } \\
\text { population } \\
(\mathrm{n}=14,787,523)\end{array}$ & $\begin{array}{l}39.6(0.5) \\
/ 42.6(0.4)\end{array}$ & $\begin{array}{l}26.1(0.4) \\
/ 25.6(0.3)\end{array}$ & $\begin{array}{l}15.3(0.3) \\
/ 15.1(0.3)\end{array}$ & $\begin{array}{l}10.3(0.2) \\
/ 9.2(0.2)\end{array}$ & $\begin{array}{l}6.6(0.2) \\
/ 5.3(0.2)\end{array}$ & $\begin{array}{l}2.1(0.1) \\
/ 2.2(0.1)\end{array}$ & 0.0165 \\
\hline $\begin{array}{l}\text { Men } \\
\qquad(n=6,443,456)\end{array}$ & $\begin{array}{l}37.4(0.7) \\
/ 37.3(0.7)\end{array}$ & $\begin{array}{l}22.8(0.6) \\
/ 23.6(0.5)\end{array}$ & $\begin{array}{l}15.6(0.5) \\
/ 17.4(0.5)\end{array}$ & $\begin{array}{l}12.5(0.4) \\
/ 11.7(0.4)\end{array}$ & $\begin{array}{l}8.5(0.3) \\
/ 7.1(0.3)\end{array}$ & $\begin{array}{l}3.1(0.2) \\
/ 3.0(0.2)\end{array}$ & 0.4675 \\
\hline $\begin{array}{l}\text { Younger aged Men } \\
\quad(n=1,990,178)\end{array}$ & $\begin{array}{l}44.1(1.3) \\
/ 38.1(1.5)\end{array}$ & $\begin{array}{l}26.8(1.0) \\
/ 26.8(1.2)\end{array}$ & $\begin{array}{l}14.9(0.8) \\
/ 17.0(1.1)\end{array}$ & $\begin{array}{l}8.3(0.5) \\
/ 10.6(0.8)\end{array}$ & $\begin{array}{l}5.5(0.5) \\
/ 5.6(0.7)\end{array}$ & $\begin{array}{l}0.4(0.1) \\
/ 1.9(0.3)\end{array}$ & 0.2059 \\
\hline $\begin{array}{l}\text { Middle aged Men } \\
\quad(n=4,453,278)\end{array}$ & $\begin{array}{l}35.8(0.8) \\
/ 36.6(0.7)\end{array}$ & $\begin{array}{l}21.9(0.7) \\
/ 20.9(0.5)\end{array}$ & $\begin{array}{l}15.9(0.6) \\
/ 17.7(0.5)\end{array}$ & $\begin{array}{l}13.5(0.6) \\
/ 12.6(0.4)\end{array}$ & $\begin{array}{l}9.3(0.5) \\
/ 8.3(0.3)\end{array}$ & $\begin{array}{l}3.7(0.3) \\
/ 3.9(0.2)\end{array}$ & 0.7652 \\
\hline $\begin{array}{l}\text { Women } \\
\qquad(n=8,344,068)\end{array}$ & $\begin{array}{l}41.2(0.6) \\
/ 46.8(0.6)\end{array}$ & $\begin{array}{l}28.6(0.5) \\
/ 27.2(0.5)\end{array}$ & $\begin{array}{l}15.1(0.4) \\
/ 13.2(0.3)\end{array}$ & $\begin{array}{l}8.7(0.3) \\
/ 7.2(0.2)\end{array}$ & $\begin{array}{l}5.1(0.2) \\
/ 3.9(0.2)\end{array}$ & $\begin{array}{l}1.4(0.1) \\
/ 1.6(0.1)\end{array}$ & 0.0003 \\
\hline $\begin{array}{l}\text { Younger aged } \\
\text { Women } \\
(n=2,754,380)\end{array}$ & $\begin{array}{l}55.9(1.0) \\
/ 56.0(1.1)\end{array}$ & $\begin{array}{l}26.9(0.7) \\
/ 25.3(0.9)\end{array}$ & $\begin{array}{l}11.8(0.5) \\
/ 11.3(0.6)\end{array}$ & $\begin{array}{l}3.1(0.2) \\
/ 5.0(0.4)\end{array}$ & $\begin{array}{l}1.5(0.1) \\
/ 1.8(0.2)\end{array}$ & $\begin{array}{l}0.9(0.2) \\
/ 0.6(0.2)\end{array}$ & 0.4899 \\
\hline $\begin{array}{l}\text { Middle aged } \\
\text { Women } \\
(n=5,589,687)\end{array}$ & $\begin{array}{l}36.9(0.7) \\
/ 38.5(0.6)\end{array}$ & $\begin{array}{l}29.1(0.6) \\
/ 29.0(0.5)\end{array}$ & $\begin{array}{l}16.1(0.5) \\
/ 15.0(0.4)\end{array}$ & $\begin{array}{l}10.2(0.4) \\
/ 9.3(0.3)\end{array}$ & $\begin{array}{l}6.1(0.3) \\
/ 5.8(0.2)\end{array}$ & $\begin{array}{l}1.5(0.1) \\
/ 2.4(0.1)\end{array}$ & 0.2629 \\
\hline $\begin{array}{l}\text { Working } \\
\quad \text { population } \\
(n=12,840,079)\end{array}$ & $\begin{array}{l}34.4(0.5) \\
/ 38.9(0.5)\end{array}$ & $\begin{array}{l}25.0(0.4) \\
/ 26.1(0.4)\end{array}$ & $\begin{array}{l}18.7(0.3) \\
/ 16.6(0.3)\end{array}$ & $\begin{array}{l}11.9(0.3) \\
/ 10.9(0.3)\end{array}$ & $\begin{array}{l}7.4(0.2) \\
/ 5.4(0.2)\end{array}$ & $\begin{array}{l}2.6(0.1) \\
/ 2.1(0.1)\end{array}$ & $<.0001$ \\
\hline $\begin{array}{l}\text { Men } \\
\qquad(n=7,153,902)\end{array}$ & $\begin{array}{l}27.6(0.6) \\
/ 29.7(0.6)\end{array}$ & $\begin{array}{l}23.0(0.5) \\
/ 24.6(0.6)\end{array}$ & $\begin{array}{l}22.2(0.5) \\
/ 19.7(0.5)\end{array}$ & $\begin{array}{l}14.3(0.4) \\
/ 14.9(0.5)\end{array}$ & $\begin{array}{l}9.4(0.3) \\
/ 8.0(0.3)\end{array}$ & $\begin{array}{l}3.4(0.2) \\
/ 3.0(0.2)\end{array}$ & 0.1410 \\
\hline $\begin{array}{l}\text { Younger aged Men } \\
\quad(n=3,544,804)\end{array}$ & $\begin{array}{l}41.0(0.9) \\
/ 34.9(1.0)\end{array}$ & $\begin{array}{l}24.6(0.7) \\
/ 25.5(1.0)\end{array}$ & $\begin{array}{l}19.2(0.6) \\
/ 18.5(0.8)\end{array}$ & $\begin{array}{l}8.9(0.4) \\
/ 12.2(0.7)\end{array}$ & $\begin{array}{l}4.8(0.3) \\
/ 6.7(0.5)\end{array}$ & $\begin{array}{l}1.4(0.2) \\
/ 2.1(0.3)\end{array}$ & 0.0458 \\
\hline $\begin{array}{l}\text { Middle aged Men } \\
\quad(n=3,609,098)\end{array}$ & $\begin{array}{l}20.8(0.8) \\
/ 20.0(0.6)\end{array}$ & $\begin{array}{l}22.2(0.8) \\
/ 23.0(0.6)\end{array}$ & $\begin{array}{l}23.8(0.8) \\
/ 21.8(0.6)\end{array}$ & $\begin{array}{l}17.1(0.7) \\
/ 20.0(0.6)\end{array}$ & $\begin{array}{l}11.8(0.6) \\
/ 10.3(0.6)\end{array}$ & $\begin{array}{l}4.4(0.4) \\
/ 4.9(0.3)\end{array}$ & 0.4969 \\
\hline $\begin{array}{l}\text { Women } \\
\qquad(n=5,686,176)\end{array}$ & $\begin{array}{l}42.8(0.7) \\
/ 50.5(0.8)\end{array}$ & $\begin{array}{l}27.5(0.6) \\
/ 27.9(0.6)\end{array}$ & $\begin{array}{l}14.2(0.4) \\
/ 12.8(0.4)\end{array}$ & $\begin{array}{l}9.0(0.3) \\
/ 5.9(0.3)\end{array}$ & $\begin{array}{l}5.0(0.2) \\
/ 2.1(0.2)\end{array}$ & $\begin{array}{l}1.6(0.1) \\
/ 0.8(0.1)\end{array}$ & $<.0001$ \\
\hline $\begin{array}{l}\text { Younger aged } \\
\text { Women } \\
(n=2,653,923)\end{array}$ & $\begin{array}{l}60.5(1.0) \\
/ 58.8(1.3)\end{array}$ & $\begin{array}{l}26.3(0.7) \\
/ 27.2(0.9)\end{array}$ & $\begin{array}{l}8.7(0.4) \\
/ 9.9(0.6)\end{array}$ & $\begin{array}{l}3.1(0.2) \\
/ 2.9(0.3)\end{array}$ & $\begin{array}{l}1.2(0.1) \\
/ 0.8(0.2)\end{array}$ & $\begin{array}{l}0.3(0.1) \\
/ 0.4(0.1)\end{array}$ & 0.8927 \\
\hline $\begin{array}{l}\text { Middle aged } \\
\text { Women } \\
(n=3,032,254)\end{array}$ & $\begin{array}{l}33.5(0.8) \\
/ 38.9(0.8)\end{array}$ & $\begin{array}{l}28.1(0.8) \\
/ 28.8(0.7)\end{array}$ & $\begin{array}{l}17.1(0.6) \\
/ 16.8(0.5)\end{array}$ & $\begin{array}{l}12.1(0.5) \\
/ 10.2(0.4)\end{array}$ & $\begin{array}{l}7.0(0.4) \\
/ 3.9(0.3)\end{array}$ & $\begin{array}{l}2.3(0.2) \\
/ 1.4(0.1)\end{array}$ & 0.0033 \\
\hline
\end{tabular}

in a previous Korean study using KNHANES 2017 data, $57.9 \%[21]$.

The results of this study showed that irregular breakfast intake ( $<5$ times per week) was closely linked to higher risk of increasing number of abnormal metabolic outcomes, especially in young men in the working population than regular breakfast intake. These results are consistent with a previous study that used the 
Table 4 Association between the number of metabolic abnormalities and irregular breakfast consumption, using negative binomial regression

\begin{tabular}{|c|c|}
\hline & $\begin{array}{l}\text { Odds Ratio ( } 95 \% \text { Confidence Interval) } \\
\text { referred regular breakfast group in each } \\
\text { category }\end{array}$ \\
\hline Total participants $(n=21,193)$ & $1.05(1.01-1.08)$ \\
\hline Men $(n=9022)$ & $1.11(1.06-1.15)$ \\
\hline Younger aged Men $(n=3065)$ & $1.14(1.06-1.23)$ \\
\hline Middle aged Men $(n=5957)$ & $1.08(1.03-1.14)$ \\
\hline Women $(n=12,171)$ & $1.02(0.98-1.07)$ \\
\hline Younger aged Women $(n=4254)$ & $1.06(0.96-1.17)$ \\
\hline Middle aged Women $(n=7917)$ & $1.01(0.96-1.07)$ \\
\hline Non-working population $(n=11,796)$ & $1.05(1.00-1.10)$ \\
\hline Men $(n=4527)$ & $1.07(1.01-1.14)$ \\
\hline Younger aged Men $(n=1066)$ & $1.12(0.97-1.29)$ \\
\hline Middle aged Men $(n=3461)$ & $1.04(0.97-1.12)$ \\
\hline Women $(n=7269)$ & $1.06(1.00-1.12)$ \\
\hline Younger aged Women $(n=2177)$ & $1.09(0.96-1.25)$ \\
\hline Middle aged Women $(n=5092)$ & $1.06(0.99-1.13)$ \\
\hline Working population $(n=9397)$ & $1.05(1.00-1.10)$ \\
\hline Men $(n=4495)$ & $1.14(1.08-1.21)$ \\
\hline Younger aged Men $(n=1999)$ & $1.15(1.03-1.27)$ \\
\hline Middle aged Men $(n=2496)$ & $1.12(1.04-1.20)$ \\
\hline Women $(n=4902)$ & $0.99(0.92-1.06)$ \\
\hline Younger aged Women $(n=2077)$ & $1.03(0.90-1.18)$ \\
\hline Middle aged Women $(n=2825)$ & $0.96(0.89-1.05)$ \\
\hline
\end{tabular}

All models are adjusted for age, educational level, income level, smoking, alcohol drinking, and physical activity

Bolds are indicated statistical significance. (p-value <0.05)

Younger population is 20-39years old

Middle-aged population is 40-59years old

KNHANES data and showed that breakfast consumption patterns were associated with a risk of metabolic outcomes [22]. Furthermore, our results corresponds with that of a review article, which reported that daily breakfast consumers were less likely to have cardiovascular disease risk factors, including elevated serum LDL-C levels, low serum HDL-C levels, and elevated blood pressure [23].

As shown in Tables 1 and 2, regular breakfast eaters had more abnormal metabolic outcomes. After stratification by age, sex, and working status, young male workers and middle-aged female workers had significant differences. Table 3 shows that regular breakfast eaters among young male workers tended to have a smaller number of metabolic abnormalities, while regular breakfast eaters in the middle-aged female worker group had a larger number of metabolic abnormalities. However, after adjusting for covariates, the significance disappeared only in the middle-aged female worker group.

According to a previous study, there was no significant association between breakfast skipping and abnormal metabolic outcomes in women. A Japanese longitudinal cohort study on factory employees showed that the average frequency of breakfast skipping was not associated with BMI and waist circumference in women [24]. Our results are consistent with those of the aforementioned study, and there are several explanations for this result. Postmenopausal status is known to be associated with abnormal metabolic outcomes. In middle-aged women, postmenopausal status has been reported to affect the outcome [25]. One study reported lower BMI and appearance-related satisfaction levels among young Korean university female students compared with European and American students [26]. This could increase the risk of eating disorders in young women, which might have affected the results.

Another cross-sectional study using the KNHANES data reported a different result and stated that the risk of abnormal metabolic outcomes increased in both men and women [27]. However, the definition of breakfast skipping in that study was different from that of our study. In that study, a breakfast skipper was defined as a 
subject who had skipped breakfast 1 day or 2 days before the survey. This definition has a limitation in the overall representation of breakfast consumption. From 2013, KNHANES changed the question regarding breakfast consumption habit from "Did you skip breakfast before 1 day or 2 days?" to "What is the average frequency of breakfast consumption per week for the past 1 year?" We considered the latter question in this study.

Herein, we have proposed several mechanisms to explain the association between abnormal metabolic outcomes and breakfast skipping. Breakfast is the very first meal of the day, which kick-starts the daily metabolism of the human body. Energy consumption will be lower than the energy requirement if breakfast is skipped before going to work. Food deprivation is known to cause a reduction in the basal metabolic rate (BMR) via compensatory metabolism [28]. The reduction in the BMR leads to the consumption of excess calories, ultimately leading to weight gain.

The time of meal consumption affects the postprandial increase in energy expenditure and blood glucose levels. A randomized repeated-measures study showed that skipping breakfast was compensated by consuming big meals at lunch. In addition, the study found that breakfast skipping increased the overall $24 \mathrm{~h}$ average blood glucose levels [29]. Another study found that breakfast skipping was associated with higher hemoglobin A1c values, which indicate poorer glycemic control [30]. A longitudinal study showed that breakfast skippers had high levels of fasting insulin [31]. Poor glycemic control is associated with high levels of glucose, insulin resistance, and high levels of fasting insulin. Insulin is known to stimulate hydroxy-methyl-glutaryl Co-A reductase activity, which plays a crucial role in the biosynthesis of cholesterol and lipids. Through these mechanisms, breakfast skipping might lead to increased fasting glucose levels, increased blood pressure, high levels of serum TG, and low levels of HDL-C.

This study observed a more significant relationship between breakfast skipping and abnormal metabolic outcomes in men in the working group than in women in all other groups. A previous study indicated that men in the working group, compared with women in the same group, had a higher risk of metabolic syndrome associated with working conditions [32]. Another study reported a significantly increased risk of metabolic syndrome in working men compared with working women [33]. The results of this study further support the idea of the working male population being vulnerable to metabolic diseases.

To the best of our knowledge, our study is the first and largest sample-sized study to explore the association between abnormal metabolic outcomes and breakfast skipping in the Korean population. Only a few studies have investigated the effect of work status on the association between breakfast skipping and abnormal metabolic outcomes. Our research indicated that the detrimental effect of breakfast skipping was evident in the working Korean male population, especially in young adults. Educating young male workers regarding the benefits of eating breakfast could be a great way to prevent further metabolic diseases.

This study identified the relationship between breakfast skipping and the number of metabolic abnormalities and proposed a novel hypothesis to explain the variable strength of association according to the stratifications. We considered stratifications, such as age and work status, which had not been used in previous studies. Work status is an important factor that affects daily metabolism. The different strengths of association according to work status implies that daily activity or stress levels might be an effect modifier of the association between breakfast skipping and abnormal metabolic outcomes.

Our study has several limitations. First, we used a selfadministered questionnaire to acquire information about breakfast consumption. This study used a self-reported questionnaire for breakfast consumption because the use of a self-reported questionnaire is common in breakfast consumption studies, and its reliability has been clinically verified in highly cited and qualified studies $[3,6]$. Moreover, the proportion of regular breakfast eaters in this study was similar to that of previous study [21]. This shows the repeatability of the questionnaire. The large sample size in our study could also reduce the effect of the error. In addition, our questionnaire was designed to include the 1-year average frequency to appropriately reflect the long-term dietary habits of the participants.

Second, considering the cross-sectional design of our study, caution must be exercised to establish a causal relationship. A longitudinal interventional study is needed to definitively unveil the exact mechanism. Third, although we stratified participants based on work status, we did not examine specific working conditions such as shift work, long working hours, manual work, and clerical work. Further analysis based on working conditions is required to determine whether breakfast skipping is an important risk factor for abnormal metabolic outcomes in the working population.

Finally, since the energy requirement for work was not quantified in this study, we could not directly compare the morning energy expenditure between the working and non-working populations. Further detailed studies are required to reveal the relationship between early morning working, breakfast skipping, and the risk of abnormal metabolic outcomes. Previous studies reported the significant association between skipping breakfast 
and diet quality $[34,35]$. Due to the lack of data on dietary quality, the quality and quantity of nutrients could not be analyzed in this study. The quality and quantity of nutrients in relation to breakfast skipping need to be clarified in future studies.

Although breakfast is considered the most important meal of the day, the percentage of regular breakfast eaters among young adults was only $37.92 \%$. This trend is in progress, accelerating the risk of metabolic outcomes among young adults. The risk is accentuated in the working population of young men, and further studies are required to clarify the association between specific working conditions (working hours or shift work), breakfast habituation, and the risk of metabolic outcomes.

\section{Conclusion}

Our study showed that breakfast skipping is associated with abnormal metabolic outcomes in the Korean male population, especially in young workers, and provided novel ideas to explain the mechanism through which breakfast skipping affects metabolic outcomes.

\section{Abbreviations}

KNHANES: Korea National Health and Nutrition Examination Survey; BMI: Body mass index; LDL-C: Low-density lipoprotein cholesterol; HDL-C: High-density lipoprotein cholesterol; TG: Triglyceride; BMR: Basal metabolic rate.

\section{Acknowledgements}

The authors thank the participants of KNHANES for the opportunity to perform this research.

\section{Authors' contributions}

$\mathrm{JH}$ and $\mathrm{WL}$ conceived and designed the study; analyzed and interpreted the data; and drafted and reviewed the manuscript. WJC and SH conducted the analyses and drafted and reviewed the manuscript. SKK interpreted the data and drafted and reviewed the manuscript. All authors have read and approved the final manuscript.

\section{Funding}

Not applicable.

\section{Availability of data and materials \\ KNHANES data are publicly available. (https://knhanes.cdc.go.kr/knhanes/eng/ index.do).}

\section{Ethics approval and consent to participate}

Data were anonymized prior to obtaining from KNHANES. All participants in the survey signed an informed consent form. The Institutional Review Board (IRB) of the Gil Medical Center, Gachon University, approved this study (IRB number: GCIRB2020-147).

\section{Consent for publication}

Not applicable.

\section{Competing interests}

The authors declare that they have no competing interests.

\section{Author details}

${ }^{1}$ College of Medicine, Gachon University, Incheon, Republic of Korea.

${ }^{2}$ Department of Occupational and Environmental Medicine, Gil Medical
Center, Gachon University College of Medicine, 21, Namdong-daero 774 beon-gil, Namdong-gu, Incheon 21565, Republic of Korea.

Received: 23 July 2020 Accepted: 24 November 2020

Published online: 07 January 2021

\section{References}

1. Asher G, Sassone-Corsi P. Time for food: the intimate interplay between nutrition, metabolism, and the circadian clock. Cell. 2015;161(1):84-92.

2. Jakubowicz D, Wainstein J, Landau Z, Raz I, Ahren B, Chapnik N, et al. Influences of breakfast on clock gene expression and postprandial glycemia in healthy individuals and individuals with diabetes: a randomized clinical trial. Diabetes Care. 2017;40(11):1573.

3. Cahill Leah E, Chiuve Stephanie E, Mekary Rania A, Jensen Majken K, Flint Alan J, Hu Frank B, et al. Prospective study of breakfast eating and incident coronary heart disease in a cohort of male US health professionals. Circulation. 2013;128(4):337-43.

4. Kaplan GA, Seeman TE, Cohen RD, Knudsen LP, Guralnik J. Mortality among the elderly in the Alameda County study: behavioral and demographic risk factors. Am J Public Health. 1987;77(3):307-12.

5. Stanton JL, Keast DR. Serum cholesterol, fat intake, and breakfast consumption in the United States adult population. J Am Coll Nutr. 1989;8(6):567-72.

6. Keski-Rahkonen A, Kaprio J, Rissanen A, Virkkunen M, Rose RJ. Breakfast skipping and health-compromising behaviors in adolescents and adults. Eur J Clin Nutr. 2003:57(7):842-53.

7. Leidy HJ, Ortinau LC, Douglas SM, Hoertel HA. Beneficial effects of a higher-protein breakfast on the appetitive, hormonal, and neural signals controlling energy intake regulation in overweight/obese, "breakfastskipping," late-adolescent girls. Am J Clin Nutr. 2013;97(4):677-88.

8. Hoyland A, Dye L, Lawton CL. A systematic review of the effect of breakfast on the cognitive performance of children and adolescents. Nutr Res Rev. 2009;22(2):220-43.

9. Szajewska H, Ruszczyński M. Systematic review demonstrating that breakfast consumption influences body weight outcomes in children and adolescents in Europe. Crit Rev Food Sci Nutr. 2010;50(2):113-9.

10. So HK, Nelson EAS, Li AM, Guldan GS, Yin J, Ng PC, et al. Breakfast frequency inversely associated with BMI and body fatness in Hong Kong Chinese children aged 9-18 years. Br J Nutr. 2011;106(5):742-51.

11. Thompson-McCormick JJ, Thomas JJ, Bainivualiku A, Khan AN, Becker AE. Breakfast skipping as a risk correlate of overweight and obesity in school-going ethnic Fijian adolescent girls. Asia Pac J Clin Nutr. 2010;19(3):372-82.

12. Cho S, Dietrich M, Brown CJP, Clark CA, Block G. The effect of breakfast type on total daily energy intake and body mass index: results from the third national health and nutrition examination survey (NHANES III). J Am Coll Nutr. 2003;22(4):296-302.

13. Purslow LR, Sandhu MS, Forouhi N, Young EH, Luben RN, Welch AA, et al. Energy intake at breakfast and weight change: prospective study of 6,764 middle-aged men and women. Am J Epidemiol. 2007;167(2):188-92.

14. Otaki N, Obayashi K, Saeki K, Kitagawa M, Tone N, Kurumatani N. Relationship between breakfast skipping and obesity among elderly: cross-sectional analysis of the HEIJO-KYO study. J Nutr Health Aging. 2017;21(5):501-4.

15. Deshmukh-Taskar P, Nicklas TA, Radcliffe JD, O'Neil CE, Liu Y. The relationship of breakfast skipping and type of breakfast consumed with overweight/obesity, abdominal obesity, other cardiometabolic risk factors and the metabolic syndrome in young adults. The National Health and nutrition examination survey (NHANES): 1999-2006. Public Health Nutr. 2013;16(11):2073-82

16. Min C, Noh H, Kang Y-S, Sim HJ, Baik HW, Song WO, et al. Skipping breakfast is associated with diet quality and metabolic syndrome risk factors of adults. Nutr Res Pract. 2011;5(5):455-63.

17. Kweon S, Kim Y, Jang M-j, Kim Y, Kim K, Choi S, et al. Data resource profile: the Korea national health and nutrition examination survey (KNHANES). Int J Epidemiol. 2014:43(1):69-77.

18. Expert Panel on Detection. Executive summary of the third report of the National Cholesterol Education Program (NCEP) expert panel on 
detection, evaluation, and treatment of high blood cholesterol in adults (adult treatment panel III). JAMA. 2486;285(19):2001.

19. Carey RM, Whelton PK. Prevention, detection, evaluation, and management of high blood pressure in adults: synopsis of the 2017 American College of Cardiology/American Heart Association hypertension guideline. Ann Intern Med. 2018;168(5):351-8.

20. Ha K, Song Y. Associations of meal timing and frequency with obesity and metabolic syndrome among Korean adults. Nutrients. 2019;11(10).

21. Jung J, Kim AS, Ko HJ, Choi HI, Hong HE. Association between breakfast skipping and the metabolic syndrome: the Korea National Health and nutrition examination survey, 2017. Medicina (Kaunas). 2020;56(8).

22. Yoo K-B, Suh H-J, Lee M, Kim J-H, Kwon JA, Park E-C. Breakfast eating patterns and the metabolic syndrome: the Korea National Health and nutrition examination survey (KNHANES) 2007-2009. Asia Pac J Clin Nutr. 2014;23(1):128-37.

23. St-Onge M-P, Ard J, Baskin Monica L, Chiuve Stephanie E, Johnson Heather M, Kris-Etherton P, et al. Meal timing and frequency: implications for cardiovascular disease prevention: a scientific statement from the American Heart Association. Circulation. 2017;135(9):e96-e121.

24. Sakurai M, Yoshita K, Nakamura K, Miura K, Takamura T, Nagasawa SY, et al. Skipping breakfast and 5-year changes in body mass index and waist circumference in Japanese men and women. Obes Sci Pract. 2017;3(2):162-70

25. Kim HM, Park J, Ryu SY, Kim J. The effect of menopause on the metabolic syndrome among Korean women. Diabetes Care. 2007;30(3):701.

26. Woo J. A survey of overweight, body shape perception and eating attitude of Korean female university students. J Exerc Nutr Biochem. 2014;18(3):287-92.

27. Park J, Kweon S, Kim Y, Jang M-J, Oh K. Dietary behaviors related to metabolic syndrome in Korean adults. Korean J Community Nutr. 2012;17(5):664-75.
28. Dulloo AG, Jacquet J. Adaptive reduction in basal metabolic rate in response to food deprivation in humans: a role for feedback signals from fat stores. Am J Clin Nutr. 1998;68(3):599-606.

29. Kobayashi F, Ogata H, Omi N, Nagasaka S, Yamaguchi S, Hibi M, et al. Effect of breakfast skipping on diurnal variation of energy metabolism and blood glucose. Obes Res Clin Pract. 2014;8(3):e249-e57.

30. Reutrakul S, Hood MM, Crowley SJ, Morgan MK, Teodori M, Knutson KL. The relationship between breakfast skipping, chronotype, and glycemic control in type 2 diabetes. Chronobiol Int. 2014;31(1):64-71.

31. Smith KJ, Gall SL, McNaughton SA, Blizzard L, Dwyer T, Venn AJ. Skipping breakfast: longitudinal associations with cardiometabolic risk factors in the childhood determinants of adult health study. Am J Clin Nutr. 2010;92(6):1316-25.

32. Vigna L, Brunani A, Brugnera A, Grossi E, Compare A, Tirelli AS, et al. Determinants of metabolic syndrome in obese workers: gender differences in perceived job-related stress and in psychological characteristics identified using artificial neural networks. Eat Weight Disord. 2019;24(1):73-81.

33. Lee W, Yeom $\mathrm{H}$, Yoon JH, Won JU, Jung PK, Lee JH, et al. Metabolic outcomes of workers according to the international standard classification of occupations in Korea. Am J Ind Med. 2016;59(8):685-94.

34. Leech RM, Worsley A, Timperio A, McNaughton SA. Understanding meal patterns: definitions, methodology and impact on nutrient intake and diet quality. Nutr Res Rev. 2015;28(1):1-21.

35. Hashimoto Y, Kaji A, Sakai R, Osaka T, Ushigome E, Hamaguchi M, et al. Skipping breakfast is associated with glycemic variability in patients with type 2 diabetes. Nutrition. 2020;71:110639.

\section{Publisher's Note}

Springer Nature remains neutral with regard to jurisdictional claims in published maps and institutional affiliations.
Ready to submit your research? Choose BMC and benefit from:

- fast, convenient online submission

- thorough peer review by experienced researchers in your field

- rapid publication on acceptance

- support for research data, including large and complex data types

- gold Open Access which fosters wider collaboration and increased citations

- maximum visibility for your research: over $100 \mathrm{M}$ website views per year

At BMC, research is always in progress.

Learn more biomedcentral.com/submissions 